\title{
Synthetic Strategies and Parameters Involved in the Synthesis of Oligodeoxyribo- and Oligoribonucleotides According to the $\boldsymbol{H}$-Phosphonate Method
}

\section{GENERAL INFORMATION AND SYNTHETIC STRATEGIES}

Although the most common method today for synthesis of oligonucleotides and their analogues is the phosphoramidite approach (Beaucage and Iyer, 1993, see also UNIT3.3), the newer $H$-phosphonate methodology can often be a preferred alternative (Garegg et al., 1985, 1986a,b,c; Froehler and Matteucci, 1986; Froehler et al., 1986). The use of $H$-phosphonates in nucleotide synthesis was pioneered by $\mathrm{Sir}$ Todd's group in Cambridge, UK, who in 1952 demonstrated the formation of $H$-phosphonate diesters in a condensation reaction of $H$-phosphonate monoesters with a protected nucleoside, promoted by diphenyl phosphorochloridate (Corby et al., 1952; Hall et al., 1957). This chemical principle was, however, not explored further; it was rediscovered three decades later (Garegg et al., 1985, 1986c) and explored for oligonucleotide synthesis (Froehler and Matteucci, 1986; Froehler et al., 1986; Garegg et al., 1986a,b,c, 1987a).

The method consists of condensing a protected nucleoside $H$-phosphonate monoester with a nucleoside in the presence of a coupling agent to produce the corresponding dinucleoside $H$-phosphonate diester. This, under various experimental conditions, can be con- verted to the dinucleoside phosphate or to a variety of backbone-modified analogues, e.g., phosphorothioates, phosphoramidates, etc. (Fig. 3.4.1).

A number of different synthetic methods can be used for the synthesis of nucleoside $H$-phosphonate building blocks. This unit discusses four alternative methods that are quite efficient, are experimentally simple, and make use of readily available reagents.

The first method consists of phosphitylating protected nucleosides with the in situ-generated tris-(1-imidazolyl)phosphine (from $\mathrm{PCl}_{3}$ and imidazole; reagent a in Fig. 3.4.2), followed by hydrolysis of the formed nucleoside diimidazolyl phosphite intermediate (Garegg et al., 1986a,c). This method (or its variants with other azoles) is probably the most widely used and can be recommended as a general basic method applicable to the preparation of both deoxyribonucleoside and ribonucleoside $H$ phosphonates (Froehler et al., 1986; Garegg et al., 1986a,b,c; Froehler, 1993). Imidazole from the first published procedure is preferred over the other azoles, because it is relatively inexpensive and forms the least reactive species.

In the second method, pyridinium $H$-pyrophosphonate (reagent b in Fig. 3.4.2), generated in situ from phosphonic acid and a condensing

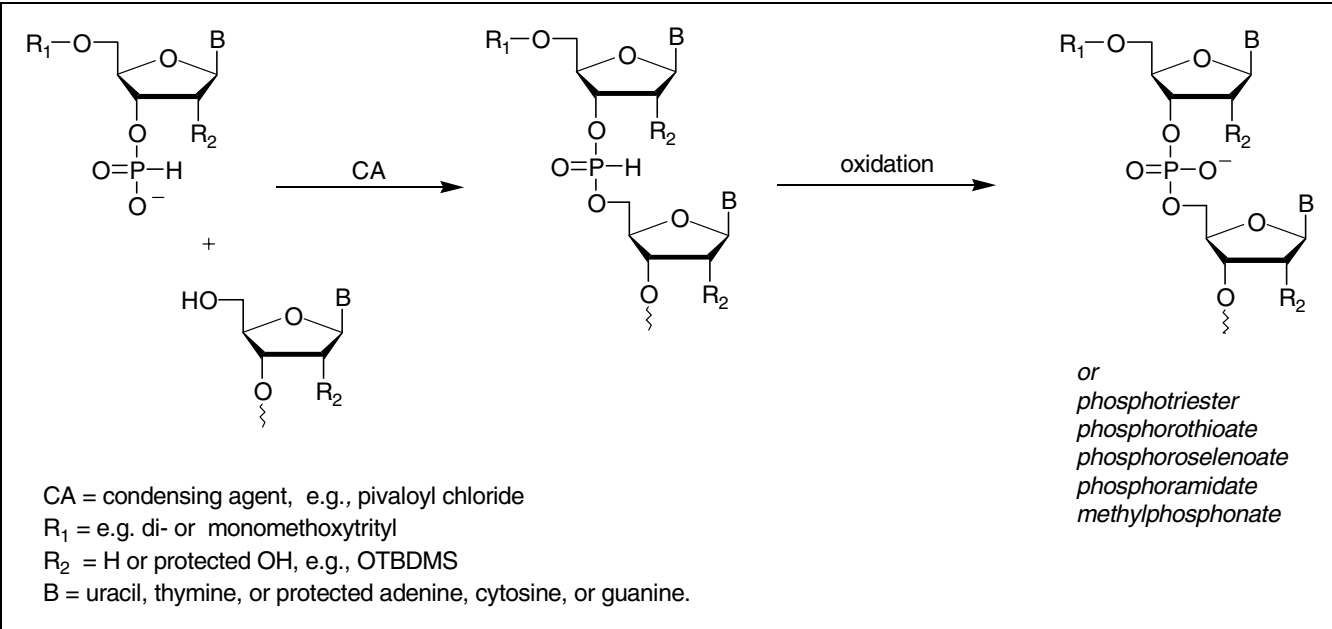

Figure 3.4.1 Condensation of protected nucleoside $H$-phosphonate monoester with a nucleoside and conversion to the dinucleoside phosphate or backbone-modified analog. 


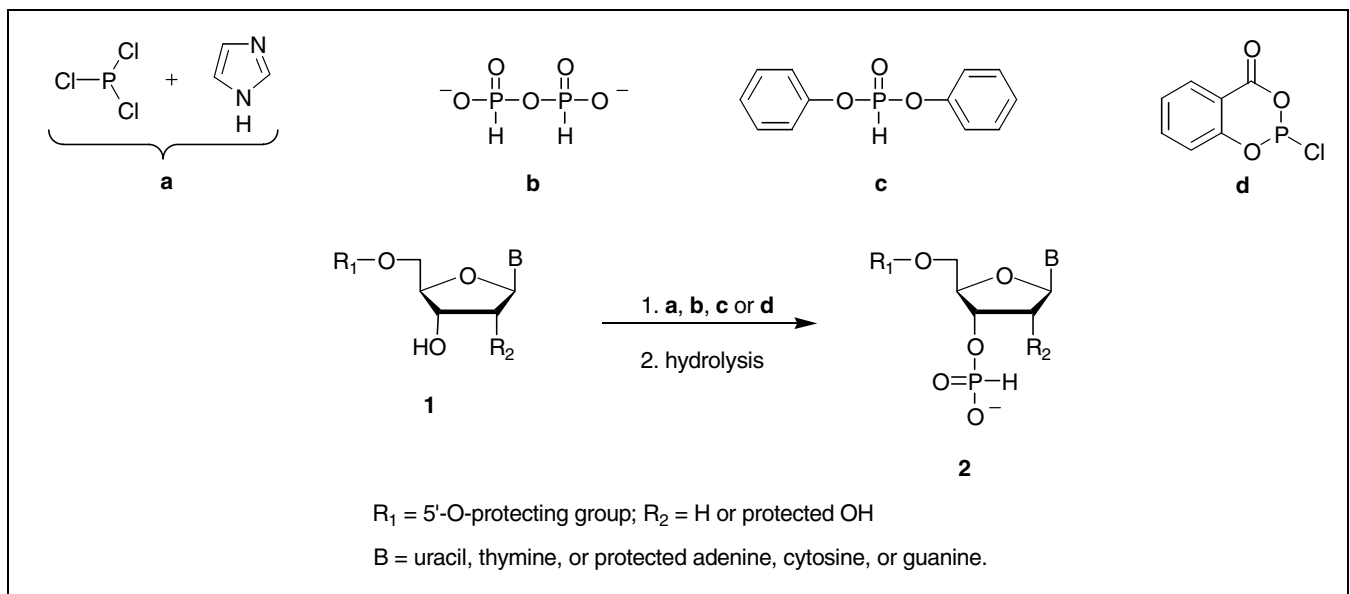

Figure 3.4.2 Reagents in the synthesis of nucleoside $H$-phosphonate building blocks.

agent, is used as phosphonylating agent (Stawinski and Thelin, 1990). This method seems to be most suitable for the preparation of deoxyribonucleoside $H$-phosphonates, because phosphonylation of the $3^{\prime}$-hydroxy group in protected ribonucleosides-e.g., in $2^{\prime}-O-t$ butyldimethylsilyl (TBDMS) protected ribonucleosides-occurs very slowly under these reaction conditions.

The third method is based on the transesterification of diphenyl $H$-phosphonate (reagent c in Fig. 3.4.2) with protected nucleosides and is a convenient approach to the preparation of nucleoside $H$-phosphonate monoesters (Jankowska et al., 1994). Diphenyl $H$-phosphonate is relatively inexpensive, commercially available, stable, and easy to handle and gives high yields of nucleoside $H$-phosphonates. Note, however, that synthesis of ribonucleoside building blocks with this method requires more basic work-up conditions, which may prevent its use with $\mathrm{N}$-protecting groups that are quite base labile.

The fourth method recommends the use of 2-chloro-4H-1,3,2-benzo-dioxaphosphinan-4 -one (salicylchlorophosphite; reagent d in Fig. 3.4.2) as the phosphitylating reagent (Marugg et al., 1986). Because salicylchlorophosphite is practically a monofunctional phosphitylating agent, it can be used in nearly stoichiometric amounts. The formation of symmetrical dinucleoside $H$-phosphonate diesters is usually negligible, but removal of by-products from the reagent can sometimes be a problem.

The condensation step of the elongation cycles (i.e., the formation of an internucleoside $H$-phosphonate linkage between a nucleoside $3^{\prime}-H$-phosphonate monoester and the supportbound $5^{\prime}$-hydroxylic component) is usually carried out in pyridine-acetonitrile mixtures. Out of the various condensing agents initially tested, pivaloyl chloride $(\mathrm{Pv}-\mathrm{Cl})$ gave the best results in automated solid-support synthesis of oligonucleotides, and it is still the most frequently used reagent. The reaction in pyridine or acetonitrile-pyridine mixtures using 2 to 5 equiv of $\mathrm{Pv}-\mathrm{Cl}$ is usually fast and goes to completion in $<1 \mathrm{~min}$. Nowadays, an array of other condensing agents (see below) is available; and these reagents can, in some instances, be superior to $\mathrm{PV}-\mathrm{Cl}$.

Oligonucleotide synthesis employing $\mathrm{H}$ phosphonates is considerably simpler than synthesis using the phosphotriester or phosphoramidite procedures. The elongation cycle includes only two chemical steps: deprotection of the terminal $5^{\prime}-\mathrm{OH}$ function of the supportbound oligonucleotide and its coupling with a nucleoside $3^{\prime}-H$-phosphonate in the presence of a condensing agent. After completion of the desired number of elongation cycles (i.e., assembly of the oligomeric chain), a single oxidation cycle is performed to convert the internucleoside $H$-phosphonate functions to phosphodiesters (or some analogue, such as phosphorothioates). Finally, the linkage between the oligomer and the support is cleaved under ammonolytic conditions, which for oligodeoxyribonucleotide and oligoribonucleotide synthesis with $2^{\prime}$ - $O$-(2-chlorobenzoyl) groups, is also the final deprotection step. $\mathrm{Pu}$ rification by standard methods is then carried out to isolate the oligonucleotides.

The H-phosphonate methodology became commercially available (for use in automated synthesizers from Applied Biosystems and Biosearch) soon after the initial reports on oligonucleotide synthesis using this approach 
were published. The research group at Applied Biosystems also introduced 1-adamantanecarbonyl chloride as an alternative to $\mathrm{Pv}-\mathrm{Cl}$ (Andrus et al., 1988). Although a capping step during oligonucleotide synthesis via $H$-phosphonate intermediates initially seemed to be superfluous, its incorporation into the synthetic protocol was shown to potentially improve the overall performance of the method. The procedure simply consists of an additional condensation step using isopropyl $H$-phosphonate after each nucleoside $H$-phosphonate coupling (Andrus et al., 1988). The use of 2-cyanoethyl $H$-phosphonate for capping was also reported, although this reagent has the disadvantage of being less accessible. The capping procedure routinely used in the phosphoramidite approach (acylation with acetic anhydride in the presence of nucleophilic catalysts more powerful than pyridine) is not compatible with $\mathrm{H}$ phosphonate chemistry, owing to the occurrence of $P$-acylation (see below).

The efficiency of each elongation step in oligonucleotide synthesis on solid support is usually high, but technical aspects of the procedure may have to be adjusted for each particular machine. The most important of these are probably the time of preactivation of the $H$-phosphonate before it reaches the solid support, the concentration of the condensing agent, and the proportion of pyridine in the solvent mixture. Some recently introduced condensing agents-e.g., bis(pentafluorophenyl) carbonate (Efimov et al., 1993) and carbonium- and phosphonium-based condensing agents (Wada et al., 1997) — however, seem to make the condensation less sensitive to these factors.

The reaction conditions for the removal of the acid-labile 5'-dimethoxytrityl or monomethoxytrityl groups (e.g., usually $1 \%$ to $2 \%$ of various haloacetic acids in an anhydrous chlorinated solvent) have been shown not to affect the integrity of the $H$-phosphonate linkages within a relevant time (Stawinski et al., 1988). The most important factors affecting the condensation and oxidation steps are discussed in more detail later in this unit.

Since the introduction of the $\mathrm{H}$-phosphonate methodology for synthesis of oligonucleotides, a number of improvements have been introduced. These involve new condensing agents and new reaction conditions. Oligodeoxynucleotide synthesis via $H$-phosphonates has been scaled up to 10 to $14 \mu \mathrm{mol}$ using only a few equivalents of monomeric building blocks relative to a support-bound oligomer per condensation (Gaffney and Jones, 1988). The $H$-phos- phonate approach was used in a cartridge-based procedure for simultaneous synthesis of multiple oligodeoxynucleotides (Seliger and Rösch, 1990). The $H$-phosphonate methodology has been used in the synthesis of a number of different oligonucleotide analogues, for example, phosphoramidates (Froehler, 1986) and phosphorothioates (Agrawal and Tang, 1990; Stein et al., 1990), including those with all- $R \mathrm{p}$ linkages (Almer et al., 1996). It has also been used in the synthesis of oligonucleotides bearing modified heterocyclic bases (Ramzaeva et al., 1997; Seela and Wei, 1997). One particularly interesting feature of oligonucleotide synthesis when using the $H$-phosphonate methodology is that it can be performed without protection of the nucleobases (Kung and Jones, 1992; Wada et al., 1997).

RNA synthesis is perhaps where the advantages of the $H$-phosphonate approach are most apparent. In the first report, the $t$-butyldimethylsilyl (TBDMS) group was used for protection of the 2'-OH and the protocol was similar to that described for DNA synthesis (Garegg et al., 1986a,b). A number of subsequent reports have differed in the choice of $2^{\prime}-\mathrm{OH}$ protection (see UNITS 2.2 and 3.5). These include the use of photolabile groups (e.g., $o$-nitrobenzyl; Tanaka et al., 1987), acid-sensitive groups (e.g., 1-(2chloro-4-methylphenyl)-4-methoxypiperidin4-yl (Ctmp); Sakatsume et al., 1989), and baselabile benzoyl derivatives (Rozners et al., 1988, 1990, 1992).

The $H$-phosphonate approach seems to be even more suited for RNA than for DNA synthesis. Potential problems-e.g., double activation (i.e., the bis-acyl phosphite formation) and $P$-acylation-are significantly suppressed when using ribonucleoside $H$-phosphonates (Stawinski et al., 1991b). Another advantage in the use of $H$-phosphonates for RNA synthesis is that the condensation reaction is virtually as fast as for DNA-synthesis and at least as efficient, even with bulky 2 '- $O$-protection. Synthesis of oligoribonucleotides of up to 50 to 60 nucleotide residues in length is readily achieved (Agrawal and Tang, 1990, Rozners et al., 1998), and the deprotection steps have been adjusted to more base-labile $N$-protection that gives better performance (Rozners et al., 1998).

The most common choice for 2'-OH protection in oligoribonucleotide synthesis is the TBDMS group (see UNIT 3.5). Studies on the stability of this group under the reaction conditions used for removal of $\mathrm{N}$-acyl groups have been carried out (Stawinski et al., 1988; Wu and Ogilvie, 1990). A conclusion (relevant not only
Synthesis of Unmodified Oligonucleotides

Current Protocols in Nucleic Acid Chemistry 
to the $H$-phosphonate approach) was that synthesis of longer RNA oligonucleotides with $2 '-O$-TBDMS protection would benefit from the use of more labile $N$-acyl-protecting groups for the heterocyclic base moieties. These were later duly introduced to the synthesis of oligoribonucleotides, both by the phosphoramidite approach (Wu and Ogilvie, 1988; Chaix et al., 1989 ) and the $H$-phosphonate method (Westman et al. 1993, 1994; Rozners et al., 1998).

Although $\mathrm{Pv}-\mathrm{Cl}$ (Froehler et al., 1986; Garegg et al., 1986a) and perhaps adamantane carbonyl chloride (Andrus et al., 1988) are the most popular condensing agents used in solidphase synthesis of oligonucleotides via the $H$ phosphonate approach, several others that may be considered for different applications have also been evaluated. These include various pivalic (Strömberg and Stawinski, 1987) or other carboxylic acid derivatives (Jäger et al., 1987), diphenyl phosphorochloridate (Hall et al., 1957; Garegg et al., 1985), 2-chloro-5,5-dimethyl-2oxo-1,3,2-dioxaphosphinane (Strömberg and Stawinski, 1987), bis(2-oxo-3-oxazolidinyl)phosphinic chloride (Strömberg and Stawinski, 1987), various dialkyl phosphorochloridates (Strömberg and Stawinski, 1987; Stawinski et al., 1988), arene sulfonic acid derivatives (Garegg et al., 1985; Jäger et al., 1987; Dan'kov et al., 1988), 1,3-dimethyl-2chloro-imidazolium chloride (Sakatsume et al., 1989, 1990), bis(pentafluorophenyl)carbonate) (Efimov et al., 1993), and a number of carbonium and phosphonium derivatives (Wada et al., 1997).

\section{PARAMETERS OF THE UNDERLYING CHEMISTRY}

\section{Activation of $\boldsymbol{H}$-phosphonate Monoesters}

The formation of an internucleoside linkage by the $H$-phosphonate approach, using acyl chlorides, proceeds via a mixed phosphonocarboxylic anhydride that reacts with the nucleosidic component to produce an $H$-phosphonate diester (Garegg et al., 1987c; Fig. 3.4.3). The order in which the reactants are added in solution synthesis with near equimolar amounts of phosphonate and nucleoside was found to be important for the efficiency of $H$-phosphonate diester formation (Garegg et al., 1985, 1986c). The coupling reaction is virtually quantitative when the condensing agent is added to a solution containing both the alcohol and the $H$-phosphonate monoester. If, on the other hand, the $H$-phosphonate is preacti- vated with a condensing agent before the addition of an alcohol, the reaction is considerably less efficient. This phenomenon was investigated and traced back to the further activation of the initially formed intermediates in the absence of an alcohol (Garegg et al., 1987c,d,e). Irrespective of the condensing agent used, tricoordinated phosphite derivatives were formed; and upon reaction with an alcohol, these species (the chemical nature of which depends on the coupling agent used) always gave various unwanted products in addition to the $H$-phosphonate diesters.

Preactivation of $H$-phosphonate monoesters with acyl chlorides produces bis-acyl phosphites (Garegg et al., 1987c). The consequence of such preactivation is that, in solution synthesis with near equimolar amounts of $\mathrm{H}$ phosphonate and alcohol, formation of phosphite triesters is unavoidable (at least to some extent). It is quite clear that such species must also be formed to some extent when the condensing agent and $H$-phosphonate are mixed before entering the column in a synthesizer. No side products that could arise from this species have so far been detected in solidphase synthesis products, but it has been shown that too long of a preactivation substantially slows down the coupling reaction, and the competing reaction of alcoholic functions with the condensing agent becomes more pronounced (Gaffney and Jones, 1988). It has also been shown that even if condensation with nucleoside bis-acyl phosphites gave the correct product on a solid support, the reaction of a bis-acyl phosphite with a nucleoside is substantially slower than that of the corresponding $H$-phosphonate which is postulated to proceed via the mixed phosphono-carboxylic anhydride (shown in Fig. 3.4.3; Garegg et al., 1987c; Efimov and Dubey, 1990).

Because an excess of $H$-phosphonate to alcohol is normally used in solid-phase synthesis, some formation of bis-acyl phosphite is tolerable. It is clear that the preactivation time should be minimized so that the mixture reaching the column has the highest ratio of mixed phosphono-carboxylic anhydride to bis-acyl phosphite as possible. Otherwise, the oligonucleotide synthesis will lose in efficiency. A preactivation time that is too long can probably not be compensated to the full extent by longer condensation time, because competing reactions become more prominent. Apart from minimizing preactivation time, it is also possible to reduce or change the amount of condensing agent (which is usually used in excess) and 


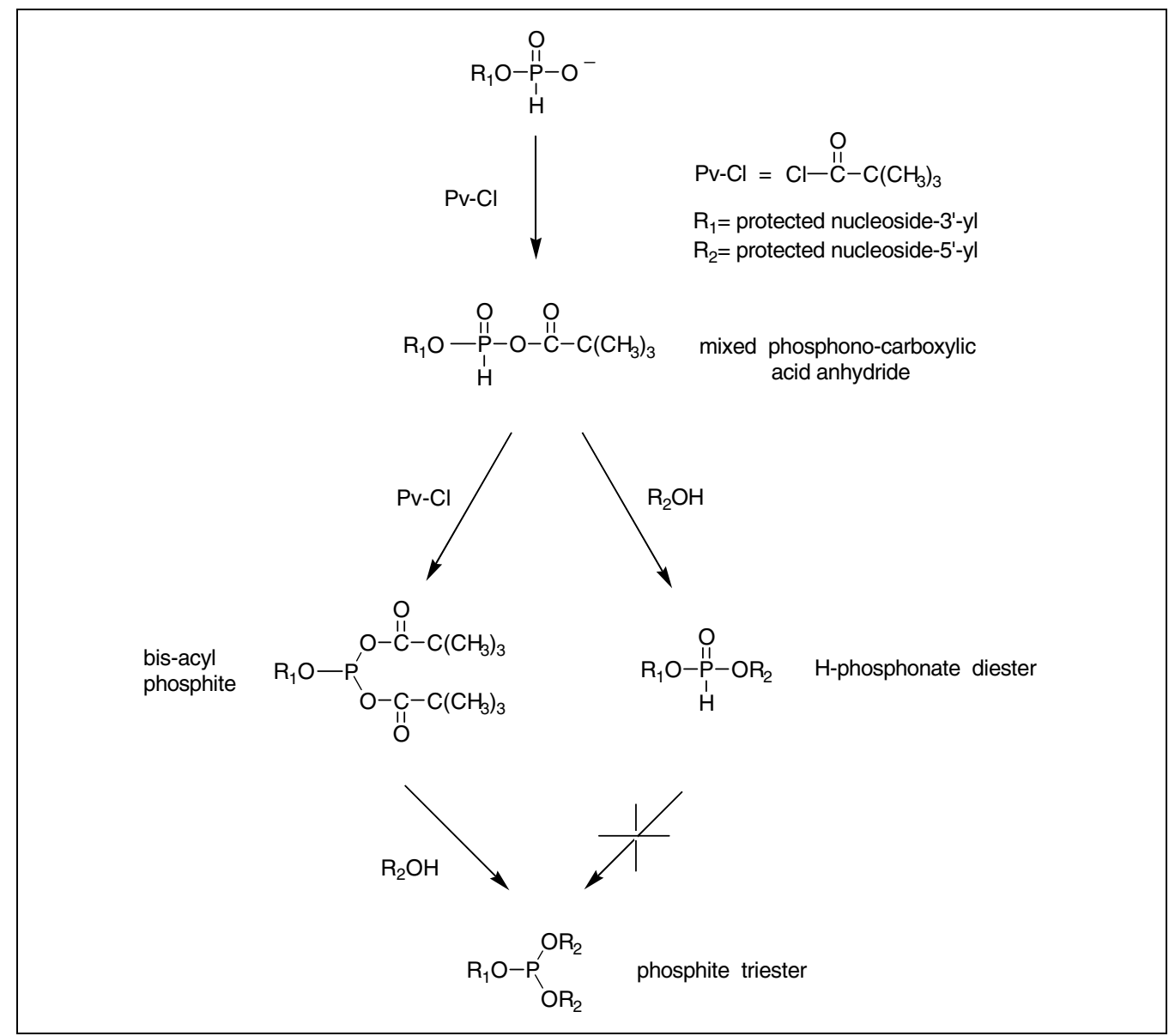

Figure 3.4.3 Activation of $H$-phosphonate monoesters.

control the reaction by varying solvent composition (see below). It is convenient to use enough condensing agent to achieve conditions that are not too moisture sensitive; but over a certain level, additional excess of acyl chloride increases the level of side reactions that may lower the coupling efficiency and overall yield.

Particularly noteworthy was the observation that $H$-phosphonate monoesters when activated in the absence of a base (like pyridine) produce only monoactivated species (Garegg et al., 1987c). Efimov and co-workers found that replacing pyridine by the slightly weaker base quinoline is sufficient to suppress the formation of bis-acyl phosphite and keep its amount $<5 \%$ to $10 \%$ within the period of time necessary for a condensation (Efimov and Dubey, 1990; Efimov et al., 1990). This novel solvent mixture (acetonitrile-quinoline, $4: 1 \mathrm{v} / \mathrm{v}$ ) was used in the synthesis of oligodeoxynucleotides up to 39 units in length (Efimov and Dubey, 1990). Although the replacement of pyridine by quinoline in oligonucleotide synthesis via the $\mathrm{H}$ phosphonate approach offers some advantages, the rate of condensation is usually lower and quinoline gives some side reactions with activated $H$-phosphonate monoesters (Stawinski et al., 1991a). The influence of base strength on preactivation of ribonucleoside $H$-phosphonates was examined, and substituted pyridines that were less basic than pyridine itself were found to suppress formation of tricoordinated species (Stawinski et al., 1991b). With these less basic pyridine derivatives, the side reactions observed with quinoline could not be detected. Steric factors are also likely to be important for double activation, because, for example, the formation of tricoordinated species is slower for $2^{\prime}-O$-TBDMS-protected ribonucleoside $H$-phosphonates than for the corresponding $2^{\prime}$-deoxyribo derivatives.

Recently, bis(pentafluorophenyl) carbonate (PFPC) was advocated as a condensing agent for $H$-phosphonate diesters formation (Efimov et al., 1993). The reagent may be as reactive as $\mathrm{Pv}-\mathrm{Cl}$ in promoting condensations and compares favorably with $\mathrm{Pv}-\mathrm{Cl}$ in regard to its reactivity toward heterocyclic bases and the 5 '-hydroxyl function of a nucleosidic component (Efimov et al., 1993). Although the re-
Synthesis of Unmodified Oligonucleotides 
agent produces double-activated species from $\mathrm{H}$-phosphonate monoesters, these are as reactive as the initially formed monoactivated intermediate. Truncated sequences are claimed to be significantly reduced compared to the protocol that uses $\mathrm{Pv}-\mathrm{Cl}$ as a condensing agent. The utility of the reagent was demonstrated in the synthesis of several oligodeoxyribonucleotides (chain length 22 to 40) and oligonucleotide-phospholipid conjugates (Efimov et al., 1993).

\section{Competing $\boldsymbol{O}$-Acylation}

Direct reaction of a condensing agent with hydroxylic functions in competition with the desired coupling reaction is another potential source of inefficiency in oligonucleotide synthesis. The suggestion that capping is important when using the $H$-phosphonate approach for oligonucleotide synthesis points to a rather low extent of acylation during the condensation step (Andrus et al., 1988; Gaffney and Jones, 1988). Self-capping by the acyl chlorides used for condensation was estimated to account for only $\sim 10 \%$ to $50 \%$ of nonphosphonylated hydroxyls (Gaffney and Jones, 1988), which represents $0.1 \%$ to $0.5 \%$ of an average coupling yield of $99 \%$. When a fourfold excess of $H$-phosphonate to nucleoside and 5 equiv $\mathrm{Pv}-\mathrm{Cl}$ were reacted for $1.5 \mathrm{~min}$, however, the total amount of $5^{\prime}$ - $O$-acylation was $\sim 0.8 \%$ (Gaffney and Jones, 1988). The extent of $O$-acylation depends heavily on reaction conditions, such as ratios and concentrations of reagents, and is likely to be substantially lower when a larger excess of $H$-phosphonate is used in conjunction with fewer equivalents of $\mathrm{Pv}-\mathrm{Cl}$.

The authors assert that the most important reason for the occurrence of competing $5^{\prime}-O$ acylation in machine-assisted synthesis is that the condensation is slowed down owing to formation of bis-acyl phosphites (see above). Acylation of 5'-OH functions has never been observed when the condensation reaction is carried out in solution without preactivation. Minimizing formation of the less reactive tricoordinated species (from preactivation) should result in higher coupling efficiency. Using fewer equivalents of condensing agent relative to $H$-phosphonate monoester is likely to be the most efficient way to decrease $5^{\prime}$ - $O$-acylation, because it will affect the rate of both $O$-acylation and bis-acyl phosphite formation. Changes in solvent composition may also be beneficial (e.g., less pyridine).

\section{$P$-Acylation}

Another side reaction that potentially can occur when using acyl chlorides as condensing agents in the $H$-phosphonate approach to oligonucleotide synthesis is the formation of acylphosphonates (Fig. 3.4.4). When the synthesis of an $\mathrm{H}$-phosphonate diester is carried out with all reactants in solution under standard conditions (approximately equimolar amounts of $H$-phosphonate and nucleosidic components together with 2 to 3 equiv of coupling agent), the reaction can be quenched long before any $P$-acylation can be detected. In solid-support oligonucleotide synthesis, however, because of iterative condensation steps, reaction of $\mathrm{H}$ phosphonate functions with the condensing agent could become a problem. Acylphosphonate formation was not detected when $H$-phosphonate diesters were exposed to 3 to 5 equiv of $\mathrm{Pv}-\mathrm{Cl}$ in pyridine for $30 \mathrm{~min}$ (Regberg et al., 1988). In a study of $P$-acylation, however, a slow conversion of a di(deoxyribonucleoside) $\mathrm{H}$-phosphonate to the corresponding pivaloylphosphonate went to completion in the presence of 5 equiv of $\mathrm{Pv}-\mathrm{Cl}$ in pyridineacetonitrile after $15 \mathrm{hr}$ at $20^{\circ} \mathrm{C}$ (Kuyl-Yeheskiely et al., 1986).

The $P$-acylation during a condensation reaction should in principle be less pronounced, owing to the inhibitory effect of pyridinium ions that are formed (Strömberg, 1987). In a condensation mixture containing 5 equiv of $\mathrm{Pv}-\mathrm{Cl}$ (in pyridine-acetonitrile $1: 1 \mathrm{v} / \mathrm{v}$ ), only $\sim 15 \%$ of the produced $H$-phosphonate diester is pivaloylated in $14 \mathrm{hr}$ (Strömberg, 1987). In the presence of bases stronger than pyridine (e.g., $N$-methylimidazole, triethylamine), the $P$-acylation is considerably faster (Strömberg, 1987). Note, however, that even if $P$-acylation occurred to some extent during oligonucleotide synthesis, this modification could not be easily carried through to the fully deprotected oligonucleotide. Oligonucleotidic chains bearing acylphosphonate modifications will be cleaved during final deprotection upon treatment with aqueous ammonia, and thus only a decrease in the yield of oligomers with the desired chain length is expected.

Reactions of protected ribonucleoside $H$ phosphonate diesters with $\mathrm{Pv}-\mathrm{Cl}$ are considerably slower than those of deoxyribonucleoside derivatives (Stawinski et al., 1991b). The presence of a bulky 2 '- $O$-TBDMS-protecting group vicinal to the $H$-phosphonate linkage in the ribonucleoside derivatives is likely to be one reason for the observed difference. In a typical condensation reaction (5 equiv of $\mathrm{Pv}-\mathrm{Cl}$ in 


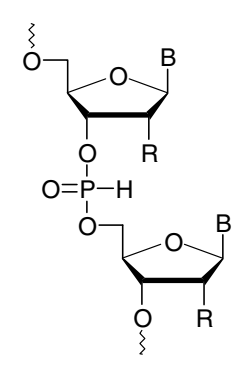

H-phosphonate diester

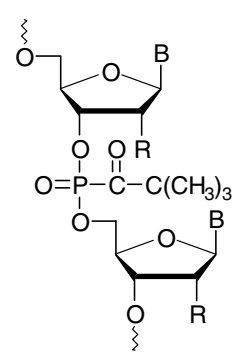

P-acylphosphonate

$\mathrm{R}=$ e.g. $\mathrm{H}$ or protected 2'-OH, e.g., OTBDMS

$\mathrm{B}=$ uracil, thymine, or protected adenine, cytosine, or guanine

Figure 3.4.4 $P$-acylation side reaction.

pyridine-acetonitrile; $1: 1 \mathrm{v} / \mathrm{v}$ ), only a few percent of the produced $H$-phosphonate diester is pivaloylated within $22 \mathrm{hr}$ (Stawinski et al., 1991b), which corresponds to the time required for 880 standard condensations reactions. As a rough estimate, a 50-residue-long 2'-OTBDMS-oligoribonucleoside $H$-phosphonate synthesized with 5 equiv of $\mathrm{Pv}-\mathrm{Cl}$ and $50 \%$ pyridine-acetonitrile $(1: 1 \mathrm{v} / \mathrm{v})$ gives truncated sequences (owing to $P$-acylation and subsequent cleavage) to a level of $0.1 \%$; it is further estimated that decreasing the excess $\mathrm{Pv}-\mathrm{Cl}$ to 3 equiv in pyridine-acetonitrile $(1: 3 \mathrm{v} / \mathrm{v})$, would only produce $\sim 0.025 \% P$-acylation. For oligodeoxyribonucleotide synthesis, the corresponding levels should be roughly $0.9 \%$ and $0.2 \%$ to $0.3 \%$ for a coupling time of $1 \mathrm{~min}$. This suggests that $P$-acylation could well be responsible for a substantial part of the observation that DNA synthesis with $H$-phosphonates, in contradistinction to oligoribonucleotide synthesis, is not quite as efficient as with phosphoramidites (when using 5 equiv of $\mathrm{Pv}-\mathrm{Cl}$ in pyridine-acetonitrile $(1: 1 \mathrm{v} / \mathrm{v}))$. It seems clear that oligodeoxyribonucleotide synthesis would benefit even more than oligoribonucleotide synthesis from reducing the excess of coupling agent and pyridine (and possibly the basicity by using a substituted pyridine; see below).

In the earlier discussion on preactivation, some advantages connected with using a slightly weaker base than pyridine as a co-solvent during the condensation step were mentioned. Such conditions are also advantageous when considering $P$-acylation. The extent of this reaction is substantially decreased when quinoline or some substituted pyridines are used instead of pyridine (Stawinski et al., 1991b). In the case of deoxyribonucleoside
$H$-phosphonate diesters, only traces of $P$-acylation can be detected ( ${ }^{31} \mathrm{P}$ NMR) after $22 \mathrm{hr}$ when 5 equiv of $\mathrm{Pv}-\mathrm{Cl}$ in quinoline-acetonitrile $(1: 3 \mathrm{v} / \mathrm{v})$ is used. For ribonucleoside $H$-phosphonate diesters, this side reaction cannot be detected even after $22 \mathrm{hr}$ under the same conditions (Stawinski et al., 1991b).

\section{Other Potential Side Reactions}

Not many studies on the extent of base modification when using the $H$-phosphonate approach for oligonucleotide synthesis have been reported. Error rates in oligodeoxynucleotides that were produced using the $H$-phosphonate methodology have been estimated by sequencing (Vasser et al., 1990). The average number of substitutions was found to be $1 / 1783$ bases. These are not necessarily caused by base modification during synthesis, but they at least indicate the maximum level of modification one may anticipate to be present in purified oligomers.

Guanosine containing dinucleoside $\mathrm{H}$ phosphonates has been reported to react with some condensing agents (Regberg et al., 1988). The guanine residue reacts with $\mathrm{Pv}-\mathrm{Cl}$ to produce a modification (most likely pivaloylation of the lactam function) that is reversed by treatment with ammonia for a much shorter time than that used for the deprotection of oligonucleotides. Because this reaction is also considerably slower than the condensation, protection of the heteroaromatic lactam system in guanine seems to be unnecessary for most applications when using $H$-phosphonate chemistry. It may, however, be advisable to analyze for base modifications if novel condensing reagents are being used.
Synthesis of Unmodified Oligonucleotides

Current Protocols in Nucleic Acid Chemistry 
Some concern has been expressed about the possibility of a partial loss of the $4,4^{\prime}$-dimethoxytrityl group from $5^{\prime}$-protected $H$-phosphonates upon standing in vials on automated synthesizers (Froehler et al., 1986; Froehler and Matteucci, 1987). It was estimated that $5^{\prime}-O$ (4,4'-dimethoxytrityl)thymidine $H$-phosphonate (triethylammonium salt $\left(\mathrm{TEAH}^{+}\right)$) dissolved in pyridine-acetonitrile $(1: 1 \mathrm{v} / \mathrm{v})$ loses $2 \%$ of its $5^{\prime}$-protection within 4 weeks (Froehler and Matteucci, 1987). Assuming a first-order reaction, this would translate to $\sim 0.05 \%$ detritylation per $1000 \mathrm{~min}$. If fresh solutions are prepared before synthesis, this can be considered a measure of the extent to which the loss of 5 -protection could be for the last added nucleotide unit in the synthesis of a 100-mer (assuming a 10-min cycle time). The replacement of $\mathrm{TEAH}^{+}$by $2,3,4,6,7,8,9,10$,-octahydropyrimido[1,2-a]azeponium $\left(\mathrm{DBUH}^{+}\right)$salts of nucleoside $H$-phosphonate monoesters was also claimed to decrease the amount of oligomers with a chain length of $n+1$ (Froehler and Matteucci, 1987) owing to suppression of partial detritylation during the condensation step. It is, therefore, conceivable that the loss of the 5 - $O$-(4-monomethoxy trityl group) frequently used in RNA synthesis should probably be negligible under these conditions. Although the detritylation step has been shown to be completely compatible with $H$-phosphonate linkages (Stawinski et al., 1988), be aware that using acid-labile $2^{\prime}-O$-protecting groups in $H$-phosphonate-based oligoribonucleotide synthesis may cause problems (Rozners et al., 1994). These can be alleviated by using the fluoride-labile $2^{\prime}-O$-TBDMS or baselabile $2^{\prime}-O$-(2-chlorobenzoyl) groups. The former group can be recommended for the synthesis of longer oligoribonucleotides, whereas the latter can be selected for shorter fragments, owing to the simplicity and cost of preparation of starting materials.

\section{The Oxidation Step}

In the phosphoramidite approach, the oxidation step is carried out after each coupling reaction (Beaucage and Caruthers, 1981; Matteucci and Caruthers, 1981; see also UNIT 3.3). It may be feasible to do so via the $H$-phosphonate method, because the condensation reaction can be accomplished in the presence of phosphodiester functions (Földesi et al., 1989; Gryaznov and Potapov, 1990). Given that $H$-phosphonate functions remain intact throughout the oligomeric chain assembly, the oxidation reaction can be carried out in a single step upon com- pletion of the synthesis. This can be advantageous for the preparation of uniformly modified oligonucleotides (e.g., elemental sulfur can produce phosphorothioate oligomers in one step from $H$-phosphonate oligonucleotides).

The final oxidation step in the $H$-phosphonate method for oligonucleotide synthesis is usually performed using a solution of iodine in aqueous pyridine (Garegg et al., 1986a,b, 1987b) or with iodine-water in the presence of another base (triethylamine, $\mathrm{N}$-methylimidazole; Froehler and Matteucci, 1986; Froehler et al., 1986). Completeness of the oxidation reaction is, of course, important for efficient synthesis of oligonucleotides. Any oligomeric chain containing an $H$-phosphonate function will be immediately cleaved in the subsequent ammonia treatment step (Hammond, 1962). Incomplete oxidation will produce shorter than expected oligomers, thus reducing the yield of the desired product. An oxidation time of $10 \mathrm{~min}$ with $2 \%$ iodine in pyridine-water (98:2) seems to be sufficient for solid-phase synthesis of 20-mers (Garegg et al., 1986a,b). Because the oxidation reaction needs to be carried out only once after chain assembly, one can extend the oxidation time (rather than using stronger bases) to ensure complete reaction within a marginal time loss. A longer oxidation time may still be advisable for longer oligonucleotides.

Competitive hydrolysis of $H$-phosphonate diesters during aqueous oxidation may be considered a potential problem. This has not yet been reported, but it may be justified to discuss this issue because $H$-phosphonate diesters are very unstable under aqueous basic conditions (Nylén, 1937; Westheimer et al., 1988). The rate constant $\left(k_{\mathrm{OH}}\right)$ for hydroxide-catalyzed oxidation with iodine was reported to be $\sim 1400$ to 4300 times larger than that for hydrolysis of a series of dialkyl $H$-phosphonates (Nylén, 1937, 1938). These values have been used to estimate that an aqueous pyridine solution containing $2 \%$ iodine should give no more than $\sim 5$ to $25 \mathrm{ppm} H$-phosphonate diester cleavage (Stawinski and Strömberg, 1993). This suggests that hydrolysis during oxidation is not a serious problem in oligonucleotide synthesis according to the $H$-phosphonate approach.

The rate of oxidation of nucleoside H-phosphonate diesters with iodine-pyridine-water can be increased by including a presilylation step (e.g., treatment with trimethylsilyl chloride) in the synthetic protocol (Hata and Sekine, 1974; Garegg et al., 1987b). This has, however, 
been used infrequently in oligonucleotide synthesis.

\section{CONCLUDING REMARKS}

Some of the advantages of the $H$-phosphonate method are as follows. The starting materials are hydrolytically stable and easy to prepare and handle. There is no need for protection of the phosphorus center. Because excess condensing reagent is normally used, the automated synthesis is not too moisture sensitive. The synthetic procedure for building blocks and oligonucleotide preparation is more time and cost effective than other methods. The activated $H$-phosphonates are more reactive and less sensitive to steric effects than the phosphoramidites; thus efficiency and rate of formation of internucleoside linkages via ribonucleoside $H$-phosphonates are at least as efficient (if not more so) than those achieved with deoxyribonucleoside $H$-phosphonates. Various oligonucleoside phosphate analogues can be obtained from one precursor by changing oxidation conditions. Because oxidation is carried out as a final synthetic step, the $H$-phosphonate approach can also be the method of choice for the preparation of uniformly modified oligonucleotides.

\section{LITERATURE CITED}

Agrawal, S. and Tang, J.-Y. 1990. Efficient synthesis of oligoribonucleotide and its phosphorothioate analogue using H-phosphonate approach. Tetrahedron Lett. 31:7541-7544.

Almer, H., Stawinski, J., and Strömberg, R. 1996. Solid support synthesis of all Rp-oligoribonucleoside phosphorothioates. Nucl. Acids Res. 24:3811-3820.

Andrus, A., Efcavitch, J.W., McBride, L.J., and Giusti, B. 1988. Novel activating and capping reagent for improved hydrogen-phosphonate DNA synthesis. Tetrahedron Lett. 29:861-864.

Beaucage, S.L. and Caruthers, M.H. 1981. Deoxynucleoside phosphoramidites - a new class of key intermediates for deoxypolynucleotide synthesis. Tetrahedron Lett. 22:1859-1862.

Beaucage, S.L. and Iyer, R.P. 1993. The synthesis of modified oligonucleotides by the phosphoramidite approach and their applications. Tetrahedron 49:6123-6194.

Chaix, C., Molko, D., and Teoule, R. 1989. The use of labile base protecting groups in oligoribonucleotide synthesis. Tetrahedron Lett. 30:71-74.

Corby, N.S., Kenner, G.W., and Todd, A.R. 1952. Nucleotides. Part XVI. Ribonucleoside-5' phosphites. A new method for the preparation of mixed secondary phosphites. J. Chem. Soc. 3669-3675.
Dan'kov, Y.V., Batchikova, N.V., Scaptsova, N.V., Besidsky, E.S., and Azhayev, A.V. 1988. H-Phosphonate solid-phase synthesis of oligodeoxyribonucleotides in syringe. Bioorg. Khim. 14:615620.

Efimov, V.A. and Dubey, I.Y. 1990. Modification of the H-phosphonate oligonucleotide synthesis on polymer support. Bioorg. Khim. 16:211-218.

Efimov, V.A., Dubey, I.Y., and Chakhmakhcheva, O.G. 1990. NMR study and improvement of $\mathrm{H}$-phosphonate oligonucleotide synthesis. $\mathrm{Nu}$ cleosides Nucleotides 9:473-477.

Efimov, V.A., Kalinkina, A.L., and Chakhmakhcheva, O.G. 1993. Dipentafluorophenyl carbonate--a reagent for the synthesis of oligonucleotides and their conjugates. Nucl. Acids Res. 21:5337-5344.

Földesi, A., Balgobin, N., Chattopadhyaya, J. 1989. Synthesis of a "branched" trinucleotide using the H-phosphonate chemistry. Tetrahedron Lett. 30:881-884.

Froehler, B.C. 1986. Deoxynucleoside H-phosphonate diester intermediates in the synthesis of internucleotide phosphate analogues. Tetrahedron Lett. 27:5575-5578.

Froehler, B.C. 1993. Oligodeoxynucleotide synthesis. H-Phosphonate approach. In Protocols for Oligonucleotides and Analogs (S. Agrawal, ed.) pp. 63-80. Humana Press, Totowa, N.J.

Froehler, B.C. and Matteucci, M.D. 1986. Nucleoside H-phosphonates: Valuable intermediates in the synthesis of deoxyoligonucleotides. Tetrahedron Lett. 27:469-472.

Froehler, B.C. and Matteucci, M.D. 1987. The use of nucleoside $\mathrm{H}$-phosphonates in the synthesis of deoxyoligonucleotides. Nucleosides Nucleotides 6:287-291.

Froehler, B.C., Ng, P.G., and Matteucci, M.D. 1986. Synthesis of DNA via deoxynucleoside H-phosphonate intermediates. Nucl. Acids Res. 14:5399-5407.

Gaffney, B.L. and Jones, R.A. 1988. Large-scale oligonucleotide synthesis by the H-phosphonate method. Tetrahedron Lett. 29:2619-2622.

Garegg, P.J., Regberg, T., Stawinski, J., and Strömberg, R. 1985. Formation of internucleotidic bond via phosphonate intermediates. Chem. Scr. 25:280-282.

Garegg, P.J., Lindh, I., Regberg, T., Stawinski, J., Strömberg, R., and Henrichson, C. 1986a. Nucleoside H-phosphonates. III. Chemical synthesis of oligodeoxyribonucleotides by the hydrogenphosphonate approach. Tetrahedron Lett. 27:4051-4054

Garegg, P.J., Lindh, I., Regberg, T., Stawinski, J., Strömberg, R., and Henrichson, C. 1986b. Nucleoside H-phosphonates. IV. Automated solid phase synthesis of oligoribonucleotides by the hydrogenphosphonate approach. Tetrahedron Lett. 27:4055-4058.
Synthesis of Unmodified Oligonucleotides 
Garegg, P.J., Regberg, T., Stawinski, J., and Strömberg, R. 1986c. Nucleoside hydrogenphosphonates in oligonucleotide synthesis. Chem. Scr. 26:59-62.

Garegg, P.J., Henrichson, C., Lindh, I., Regberg, T., Stawinski, J., and Strömberg, R. 1987a. Automated solid phase synthesis of DNA and RNA fragments by the hydrogenphosphonate approach. In Biophosphates and Their Analogues-Synthesis, Structure, Metabolism and Activity (K.S. Bruzik and W.J. Stec, eds.) pp. 93-98. Elsevier/North-Holland, Amsterdam.

Garegg, P.J., Regberg, T., Stawinski, J., and Strömberg, R. 1987b. Nucleoside H-phosphonates. VII. Studies on the oxidation of nucleoside hydrogenphosphonate esters. J. Chem. Soc. Perkin Trans. I:1269-1273.

Garegg, P.J., Regberg, T., Stawinski, J., and Strömberg, R. 1987c. Nucleosides H-phosphonates. V. The mechanism of hydrogenphosphonate diester formation using acyl chlorides as coupling agents in oligonucleotide synthesis by the hydrogenphosphonate approach. Nucleosides Nucleotides 6:655-662.

Garegg, P.J., Stawinski, J., et al. 1987d. Nucleoside H-phosphonates. VI. Reaction of nucleoside hydrogenphosphonates with arenesulfonyl chlorides. J. Chem. Soc. Perkin Trans. II:1209-1214.

Garegg, P.J., Stawinski, J., and Strömberg, R. 1987e. Nucleoside H-phosphonates. VIII. activation of hydrogenphosphonate monoesters by chlorophosphates and aryl sulfonyl derivatives. J. Org. Chem. 52:284-287.

Gryaznov, S.M. and Potapov, V.K. 1990. New approach in synthesis of natural and modified oligodeoxyribonucleotides by H-phosphonate method. Bioorg. Khim. 16:1419-1422.

Hall, R.H., Todd, A., and Webb, R.F. 1957. Nucleotides. Part XLI. Mixed anhydrides as intermediates in the synthesis of dinucleoside phosphates. J. Chem. Soc. 3291-3296.

Hammond, P.R. 1962. A simple preparation of alkyl ammonium phosphonates and some comments on the reaction. J. Chem. Soc. 25212522.

Hata, T. and Sekine, M. 1974. Silyl phosphites. I. The reaction of silyl phosphites with diphenyl disulphides. Synthesis of S-phenyl nucleoside phosphorothioates. J. Am. Chem. Soc. 96:73637364.

Jankowska, J., Sobkowski, M., Stawinski, J., and Kraszewski, A. 1994. Studies on aryl H-phosphonates. I. Efficient method for the preparation of deoxyribo- and ribonucleoside 3'-H-phosphonate monoesters by transesterification of diphenyl H-phosphonate. Tetrahedron Lett. 35:3355-3358.

Jäger, A., Charubala, R., and Pfleiderer, W. 1987. Synthesis and characterization of deoxy and ribo H-phosphonate dimers. Nucl. Acids Symp. Ser. 18:197-200.
Kung, P.-P. and Jones R.A. 1992. H-phosphonate DNA synthesis without amino protection. Tetrahedron Lett. 33:5869-5872.

Kuyl-Yeheskiely, E., Spierenberg, M., van den Elst, H., Tromp, M., van der Marel, G.A., and van Boom, J.H. 1986. Reaction of pivaloyl chloride with internucleosidic H-phosphonate diesters. Recl. Trav. Chim. Pays-Bas 105:505-506.

Marugg, J.E., Tromp, M., Kuyl-Yeheskiely, E., van der Marel, G.A., and van Boom, J.H. 1986. A convenient and general approach to the synthesis of properly protected d-nucleoside- $3^{\prime}$ hydrogenphosphonates via phosphite intermediates. Tetrahedron Lett. 27:2661-2664.

Matteucci, M.D. and Caruthers, M.H. 1981. Synthesis of deoxyoligonucleotides on a polymer support. J. Am. Chem. Soc. 103:3185-3191.

Nylén, P. 1937. Die Kinetik der Verseifung von Dialkylphosphiten. II. Allgemeine Säure- und Basekatalyse bei der Hydrolyse von Diäthylphosphit. Svensk Kem. Tidskr. 49:79-96.

Nylén, P. 1938. Die Säure- und Basekatalyse bei der Reaktion zwischen Dialkylphosphiten und Jod. Z. Anorg. Allg. Chem. 235:161-182.

Ramzaeva, N., Mittelbach, C., and Seela, F. 1997. 7-deazaguanine DNA: Oligonucleotides with hydrophobic or cationic side chains. Helv. Chim. Acta 80:1809-1822.

Regberg, T., Stawinski, J., and Strömberg, R. 1988. Nucleoside H-phosphonates. IX. Possible sidereactions during hydrogenphosphonate diester formation. Nucleosides Nucleotides 7:23-35.

Rozners, E., Kumpins, V., Rekis, A., and Bizdena, E. 1988. Solid phase synthesis of oligoribonucleotides by the H-phosphonate method using 2'-O-benzoyl protective group. Bioorg. Khim. 14:1580-1582.

Rozners, E., Rekis, A., Kumpins, V., and Bizdena, E. 1990. Synthesis of oligoribonucleotides by the H-phosphonate method using base-labile 2' O-protecting groups. II. Some aspects of use of $2^{\prime}$ - $O$-benzoyl and anisoyl protecting groups. Bioorg. Khim. 16:1531-1536.

Rozners, E., Renhofa, R., Petrova, M., Popelis, J., Kumpins, V., and Bizdena, E. 1992. Synthesis of oligoribonucleotides by the H-phosphonate approach using base labile 2 '-O-protecting groups. 5 . Recent progress in development of the method. Nucleosides Nucleotides 11:1579. 1593.

Rozners, E., Westman, E., and Strömberg, R. 1994. Evaluation of 2'-hydroxyl protection in RNAsynthesis using the H-phosphonate approach. Nucl. Acids Res. 22:94-99.

Rozners, E., Westman, E., Stawinski, J., Garegg, P.J., Bizdena, E., and Strömberg, R. 1998. Oligoribonucleotide synthesis with H-phosphonates. An improved procedure with base labile $\mathrm{N}$-acyl protection and two alternative 2 '-O-protecting groups. Manuscript in preparation. 
Sakatsume, O., Ohtsuka, M., Takaku, H., and Reese, C.B. 1989. Solid phase synthesis of oligoribonucleotides using the 1-[2-chloro-4methyl)phenyl]-4-methoxypiperidin-4-yl (Ctmp) group for the protection of the 2 '-hydroxy functions and the H-phosphonate approach. Nucl. Acids Res. 17:3689-3696.

Sakatsume, O., Yamane, H., Takaku, H., and Yamamoto, N. 1990. Use of new phosphonylating and coupling agents in the synthesis of oligodeoxyribonucleotides via the H-phosphonate approach. Nucl. Acids Res. 18:3327-3331.

Seela, F. and Wei, C.F. 1997. 7-Deazaisoguanine quartets: Self-assembled oligonucleotides lacking the Hoogsteen motif. Chem. Commun. 18691870.

Seliger, H. and Rösch, R. 1990. Simultaneous synthesis of multiple oligonucleotides using nucleoside H-phosphonate intermediates. DNA Cell Biol. 9:691-696.

Stawinski, J. and Strömberg, R. 1993. H-phosphonates in oligonucleotide synthesis. Trends Org. Chem. 4:31-67.

Stawinski, J. and Thelin, M. 1990. Nucleoside Hphosphonates. XI. A convenient method for the preparation of nucleoside $\mathrm{H}$-phosphonates. $\mathrm{Nu}$ cleosides Nucleotides 9:129-135.

Stawinski, J., Strömberg, R., Thelin, M., and Westman, E. 1988. Studies on the t-butyldimethylsilyl group as 2'-O-protection in oligoribonucleotide synthesis via the $\mathrm{H}$-phosphonate approach. $\mathrm{Nucl}$. Acids Res. 16:9285-9298.

Stawinski, J., Strömberg, R., and Westman, E. 1991a. Ribonucleoside H-phosphonates. Pyridine vs quinoline--influence on condensation rate. Nucleosides Nucleotides 10:519-520.

Stawinski, J., Strömberg, R., and Westman, E. 1991b. Studies on reaction conditions for ribonucleotide synthesis via the H-phosphonate approach. Nucl. Acids Symp. Ser. 24:228.

Stein, A., Iversen, P.L., Subasinghe, C., Cohen, J.S., Stec, W.J., and Zon, G. 1990. Preparation of 35S-labelled polyphosphorothioate oligodeoxyribonucleotides by use of hydrogenphosphonate chemistry. Anal. Biochem. 188:11-16.

Strömberg, R. 1987. Nucleoside H-phosphonates. Chemical studies directed towards oligonucleotide synthesis. Chem. Commun. Stockholm Univ. 1:1-54.
Strömberg, R. and Stawinski, J. 1987. Evaluation of some new condensing reagents for hydrogenphosphonate diester formation. Nucl. Acids Symp. Ser. 18:185-188.

Tanaka, T., Tamatsukuri, S., and Ikehara, M. 1987. Solid phase synthesis of oligoribonucleotides using the o-nitrobenzyl group for 2'-hydroxyl protection and H-phosphonate chemistry. $\mathrm{Nucl}$. Acids Res. 15:7235-7248.

Vasser, M., Ng, P.G., Jhurani, P., and Bischofberger, N. 1990. Error rates in oligodeoxynucleotides synthesized by the H-phosphonate method. Nucl. Acids Res. 18:3089.

Wada, T., Sato, Y., Honda, F., Kawahara, S., and Sekine, M. 1997. Chemical synthesis of oligodeoxyribonucleotides using $\mathrm{N}$-unprotected $\mathrm{H}$ phosphonate monomers and carbonium and phosphonium condensing reagents: O-Selective phosphonylation and condensation. J. Am. Chem. Soc. 119:12710-12721.

Westheimer, F.H., Huang, S., and Covitz, F. 1988. Rates and mechanisms of hydrolysis of esters of phosphorous acid. J. Am. Chem. Soc. 110:181185.

Westman, E., Stawinski, J., and Strömberg, R. 1993. RNA-synthesis using H-phosphonates. Synchronizing 2'-OH and N-protection. Collect. Czech. Chem. Commun. 58:236-237.

Westman, E., Sigurdsson, S., Stawinski, J., and Strömberg, R. 1994. Improving the H-phosphonate approach to oligoribonucleotide synthesis. Nucl. Acids Symp. Ser. 31:25.

Wu, T. and Ogilvie K.K. 1988. N-Phenoxyacetylated guanosine and adenosine phosphoramidites in the solid phase synthesis of oligoribonucleotides: synthesis of a ribozyme sequence. Tetrahedron Lett. 29:4249-4251.

Wu, T. and Ogilvie, K.K. 1990. A study on the alkylsilyl groups in oligoribonucleotide synthesis. J. Org. Chem. 55:4717-4724.
Contributed by Roger Strömberg
Karolinska Institute
Stockholm, Sweden
Jacek Stawinski
Stockholm University
Stockholm, Sweden

Synthesis of Unmodified Oligonucleotides 